\title{
Changes of cardiac biomarkers after ultradistance and standard-distance triathlon
}

\author{
Chan Ho Park', Yi-Sub Kwak ${ }^{2 *}$ \\ ${ }^{1}$ Sports Science Center, Pukyong National University, Busan, Korea \\ ${ }^{2}$ DEU Exe-Physio Lab, Department of Physical Education, Dong-Eui University, Busan, Korea
}

Triathlon is becoming more popular sport due to recognition of the positive effects of triathlon. Generally, participants in these strenuous activities are well considered as healthy individuals pursuing a healthy lifestyle. However, there is still controversy on the beneficial effects of prolonged exercise training and endurance sport events. The duration of exercise required to elicit cardiac dysfunction and the mechanisms underlying this phenomenon have not been fully elucidated. There are only limited data in literature for exercise-induced changes of cardiac and muscle damage biomarkers in athletes participating in different triathlon distances. Monitoring cardiac and muscle damage biomarkers in triathletes participating in different triathlon distances will help researchers, coaches, and athletes better understand how to design training cycles minimizing overtraining and injury risk. Therefore, the purpose of the present study was to examine for evidence of blood biomarkers during triathlon events of two different distances such as standard- and ultradistance triathlon in male triathletes. The results of the present study showed that ultradistance showed greater muscle damage markers such as creatine kinase, myoglobin and lactate dehydrogenase than standard-distance. We also found that the distance of triathlon did not lead to an increase of troponin $\mathrm{T}$ in male triathletes.

Keywords: Endurance sport events, Cardiac dysfunction, Muscle damage biomarkers, Triathlon, Troponin T

\section{INTRODUCTION}

Aerobic exercise is well recognized with a lower risk of cardiac disease. Recently, many people participate in a variety of aerobic activities such as triathlon and marathon. Acute and endurance exercise substantially increase in maximum oxygen consumption, cardiac output, stroke volume, and systolic blood pressure. Among these, triathlon is becoming more popular sports due to recognition of the positive effects of these. Generally, participants in these strenuous activities are well considered as healthy individuals pursuing a healthy lifestyle. However, there is still controversy on the beneficial effects of prolonged training and endurance sport events. Prolonged strenuous exercise leads to an elevation of cardiac specific biomarkers such as troponin $\mathrm{T}$ (cTnT), troponin I (cTnI), MB-creatine kinase (CKMB), and N-terminal pro brain natriuretic peptide (NT-pro BNP) in healthy endurance athletes (Park et al., 2014). c'Tn'T and c'TnI are highly sensitive and specific markers to detect myocardial damage in the presence of peripheral muscle damage (Thygesen et al., 2007). After myocardial damage, troponins are released from cardiomyocytes and are detected within 3-10 hr in peripheral blood (Traiperm et al., 2012). Myoglobin and creatine kinase $(\mathrm{CK})$ indicating exercise-induced skeletal muscle damage are well found after triathlon events in triathletes (Park et al., 2014). Monitoring of CK obtains an information about the state of the muscle and provides an information on the physical status of the athlete (Brancaccio et al., 2007) especially during the endurance events. For the precise detection of myocardial muscle damage, more cardiac specific biomarkers such as CKMB and cardiac troponins have been identified (Agewall et al., 2011; Thygesen et al., 2007).

Generally, full-distance triathlon consists of a $3.8-\mathrm{km}$ swimming, 180.2-km cycling, and 42.2-km running and a half-distance tri-
${ }^{\star}$ Corresponding author: Yi-Sub Kwak (D) https://orcid.org/0000-0003-4545-7250 Department of Physical Education, College of Arts and Sports Science,

Dong-Eui University, 176 Eomgwang-ro, Busanjin-gu, Busan 47340, Korea E-mail: ysk2003@deu.ac.kr

Received: February 23, 2019 / Accepted: March 27, 2019
This is an Open Access article distributed under the terms of the Creative Commons Attribution Non-Commercial License (http://creativecommons.org/licenses/by-nc/4.0/) which permits unrestricted non-commercial use, distribution, and reproduction in any medium, provided the original work is properly cited. 
athlon consists of a 1.9-km swimming, 90-km cycling, and 21-km running. This distance is known as ultradistance (UD) triathlon. Olympic triathlon consists of a $1.5-\mathrm{km}$ swimming, 40-km cycling, and 10-km running and is known as standard-distance (SD) triathlon. It may be the unique physiological demand during triathlon events of different distances. Previous studies investigated blood biomarkers and cardiac dysfunction following ultratriathlon (Areces et al., 2015; Danielsson et al., 2017; Whyte et al., 2000) and also evaluated cardiac biomarkers following standard triathlon (Park et al., 2014). The duration of exercise required to elicit cardiac dysfunction and the mechanisms underlying this phenomenon have not been fully elucidated. There are only limited data in literature for exercise-induced changes of cardiac and muscle damage biomarkers in athletes participating in different triathlon distances. Monitoring of cardiac and muscle damage biomarkers in triathletes participating in different triathlon distances will help researchers, coaches, and athletes to reduce overtraining and even sports injury (Lee et al., 2017). Therefore, the purpose of the present study was to examine for evidence of blood biomarkers during triathlon events of two different distances such as SD and UD in male triathletes.

\section{MATERIALS AND METHODS}

\section{Subject and design}

Experimental design was implemented with a pre, post, and recovery measurement to determine the changes of blood biomarkers after events of different triathlon distances in amateur triathletes. This study received ethical approval (DIRB-201706-HR-R017) from University of Dong-Eui Institutional Review Board. A signed informed consent was obtained from all participants.

Blood was taken from an antecubital vein. Three days before the race, baseline blood samples were collected after an overnight fasting (8-10 hr). Postrace blood samples were collected in the supine position immediately and three hours after following the triathlon.

Eight healthy amateur male triathletes (age, $39.12 \pm 4.61$ years) who regularly participated in triathlon training and race were recruited from triathletes. All triathletes participated in standardand ultratriathlon race and also completed two races. During the study, all athletes consented to exercise normally, maintain normal diet, weight status, and avoid the use of medications known to affect exercise performance time, cardiac markers, and energy substrates responses. Physical characteristics of the participants are given in Table 1.
Table 1. Characteristics of subjects and performance

\begin{tabular}{lc}
\hline Characteristic & Value \\
\hline Age $(\mathrm{yr})$ & $39.12 \pm 4.61$ \\
Weight $(\mathrm{kg})$ & $66.87 \pm 5.6$ \\
Height $(\mathrm{m})$ & $176.0 \pm 2.9$ \\
Career $(\mathrm{yr})$ & $3.25 \pm 1.16$ \\
UD finish time (min) & $817.1 \pm 139.7$ \\
SD finish time (min) & $162.1 \pm 12.7$ \\
\hline
\end{tabular}

Values are presented as mean \pm standard deviation.

UD, ultradistance; SD, standard-distance.

\section{Measurements}

Blood samples were taken from an antecubital vein 3 days before the race, immediately, and three hour after the complete race. Blood samples for plasma analyses were collected into 5- to 8-mL vacutainer tubes containing lithium heparin. Samples were frozen at $-80^{\circ} \mathrm{C}$ for later analysis. Blood samples were assayed for markers of muscle damage, hsTnT, and energy substrates by assay kit and spectrophotometry. CK activity and CKMB activity were analyzed with Modular System P (Roche Diagnostics GmbH, Mannheim, Germany). Myoglobin was analyzed with Elecsys Modular Analytics E170 (Roche Diagnostics GmbH). cTnT was analyzed with Elecsys Modular Analytics E170 (Roche Diagnostics GmbH). Although the detection limit for cTnT is $0.01 \mathrm{ng} / \mathrm{mL}$, the recommended diagnostic threshold for myocardial damage is $>0.3 \mathrm{ng} / \mathrm{mL}$.

\section{Statistical analysis}

For statistical calculation, the IBM SPSS ver. 18.0 (IBM Co., Armonk, NY, USA) were used. All data are expressed as the mean \pm standard deviation. The differences between baseline values and the postexercise values were established for all biomarkers using the Students $t$-test. Comparison between time points for each group was performed using independent $t$-test. For all statistical tests, the threshold for statistical significance was set at $P \leq$ 0.05 .

\section{RESULTS}

Table 2 shows the changes of cardiac biomarkers after different triathlon distances. CK levels were significantly higher at postrace than at prerace in all distance $(P<0.001)$. CK levels were also significantly higher in UD than in SD at post and recovery among the groups $(P<0.001)$. Myoglobin levels were significantly higher at postrace than at prerace in $\mathrm{UD}(P<0.001)$ and in $\mathrm{SD}(P<0.01)$. $\mathrm{CKMB}$ levels were significantly higher at postrace than at prerace in all distance $(P<0.001)$. However, $\mathrm{CKMB}$ levels were not sig- 
Table 2. Changes in biomarkers of skeletal and cardiac muscle damage before, immediately after and $48 \mathrm{hr}$ after the race

\begin{tabular}{llccc}
\hline Variable & \multicolumn{2}{c}{ Pre } & Post & Recovery \\
\hline CK $(\mathrm{mg} / \mathrm{dL})$ & UD & $189.67 \pm 34.84$ & $1,128.07 \pm 247.62^{* *}$ & $388.83 \pm 62.17$ \\
& SD & $168.00 \pm 37.39$ & $466.53 \pm 181.60^{* *}$ & $246.08 \pm 83.40$ \\
& P-value & 0.928 & 0.000 & 0.002 \\
CKMB $(\mathrm{mg} / \mathrm{dL})$ & UD & $2.39 \pm 0.66$ & $9.07 \pm 2.51^{* *}$ & $3.53 \pm 0.95$ \\
& SD & $2.09 \pm 0.65$ & $7.48 \pm 2.81^{* *}$ & $2.65 \pm 0.84$ \\
& $P$-value & 0.393 & 0.252 & 0.072 \\
Myoglobin & UD & $38.22 \pm 4.68$ & $1,467.47 \pm 1,002.56^{* * *}$ & $68.97 \pm 26.96$ \\
$(\mu \mathrm{g} / \mathrm{L})$ & SD & $27.46 \pm 4.33$ & $369.19 \pm 275.07^{*}$ & $36.42 \pm 9.05$ \\
& $P$-value & 0.000 & 0.010 & 0.006 \\
LDH (mg/dL) & UD & $262.40 \pm 23.11$ & $552.48 \pm 51.64^{* *}$ & $323.08 \pm 49.24$ \\
& SD & $228.45 \pm 32.39$ & $294.74 \pm 24.99^{*}$ & $248.00 \pm 32.02$ \\
& $P$-value & 0.030 & 0.000 & 0.003 \\
CTnT (ng/mL) & UD & $0.01 \pm 0.00$ & $0.02 \pm 0.01$ & $0.03 \pm 0.03$ \\
& SD & $0.01 \pm 0.00$ & $0.01 \pm 0.01$ & $0.03 \pm 0.02$ \\
& $P$-value & - & 0.445 & 0.873 \\
\hline
\end{tabular}

Values are presented as mean \pm standard deviation.

UD, ultradistance; SD, standard-distance; CK, creatine kinase; CKMB, MB-creatine kinase; LDH, lactate dehydrogenase; $c T n T$, troponin T.

${ }^{*} P \leq 0.01 .{ }^{*} P \leq 0.001$

nificantly different among distance. Myoglobin levels were also significantly higher in UD than in SD at post and recovery among distance $(P<0.01)$. LDH levels were significantly higher at postrace than at prerace in UD $(P<0.001)$ and in SD $(P<0.01)$. LDH levels were also significantly higher in UD than in SD at post $(P<$ $0.001)$ and recovery $(P<0.01)$ among the groups. cTnT levels were not significantly different among the groups and time points. Table 3 shows the changes of cortisol and free fatty acid (FFA) after different triathlon distances. Cortisol levels were significantly higher at postrace than at prerace in all groups $(P<0.001)$. Cortisol levels were also significantly higher in UD than in SD at post $(P<0.01)$, but were not significantly different among the groups at recovery. FFA levels were significantly higher at postrace than at prerace in all distance $(P<0.01)$. FFA levels were also significantly higher in UD than in SD among the groups at post $(P<$ $0.01)$ and recovery $(P<0.05)$.

\section{DISCUSSION}

The previous studies have demonstrated that the duration of triathlon are the main factors affecting physiological responds (Olcina et al., 2018; Suzuki et al., 2006). In this study, we investigated the acute effects on biomarkers related to skeletal and cardiac muscle damage in a sample of male triathletes according to dif-
Table 3. Changes in biochemical variables before, immediately after and $48 \mathrm{hr}$ after the race

\begin{tabular}{clccc}
\hline Variable & & Pre & Post & Recovery \\
\hline Cortisol & UD & $23.04 \pm 2.94$ & $30.52 \pm 6.14^{* *}$ & $14.18 \pm 2.65$ \\
$(\mu \mathrm{g} / \mathrm{dL})$ & SD & $20.97 \pm 4.57$ & $25.08 \pm 3.39^{* *}$ & $13.26 \pm 4.22$ \\
& $P$-value & 0.300 & 0.046 & 0.610 \\
FFA (mmol/L) & UD & $303.12 \pm 101.79$ & $2,346.50 \pm 475.69^{* *}$ & $248.25 \pm 47.06$ \\
& SD & $352.00 \pm 101.99$ & $1,703.25 \pm 196.82^{* *}$ & $338.25 \pm 98.01$ \\
& P-value & 0.354 & 0.003 & 0.035 \\
\hline
\end{tabular}

UD, ultradistance; SD, standard-distance; FFA, free fatty acid.

${ }^{*} P \leq 0.001$.

ferent distance triathlon such as ultradistance and standard-distance. CK and myoglobin indicating exercise-induced skeletal muscle damages were found after triathlon events in triathletes (Park et al., 2014). Lactate dehydrogenase (LDH) levels can be increased by UD and are generally used as markers of muscle and liver damage (Suzuki et al., 2006). The results of the present study showed that UD showed greater muscle damage markers such as CK, myoglobin and LDH than SD.

Prolonged strenuous exercise leads to an elevation of cardiac specific biomarkers such as $\mathrm{CTnT}$ and CKMB in healthy endurance athletes (Park et al., 2014). CKMB also showed increases after different distance races compared with the pre levels. In contrast to these elevated markers, cTnT levels were not increases after different distance races compared with the pre levels. Though previous studies showed c $\operatorname{Tn} \mathrm{T}$ release in marathon or ultramarathon runners (Jassal et al., 2009), there was no release of cTnT after different distance races compared with the prelevels in our study. Some studies showed that the relative intensity is correlated with cTnT release such that higher intensity but shorter duration leads to a greater release (Fu et al., 2009; Stewart et al., 2016). Other studies suggested that there was no cardiac dysfunction after an Ironman-distance triathlon (Leischik and Spelsberg, 2014). We also found that the distance of triathlon did not lead to an increase of cTnT in male triathletes. Further study on specific mechanism of cardiac dysfunction is needed in this area.

\section{CONFLICT OF INTEREST}

No potential conflict of interest relevant to this article was reported.

\section{ACKNOWLEDGMENTS}

This work was supported by the Ministry of Education of the 
Republic of Korea and the National Research Foundation of Korea (NRF-2016S1A5B5A07917324).

\section{REFERENCES}

Agewall S, Giannitsis E, Jernberg T, Katus H. Troponin elevation in coronary vs. non-coronary disease. Eur Heart J 2011;32:404-411.

Areces F, González-Millán C, Salinero JJ, Abian-Vicen J, Lara B, GalloSalazar C, Ruiz-Vicente D, Del Coso J. Changes in serum free amino acids and muscle fatigue experienced during a half-ironman triathlon. PLoS One 2015;10:e0138376.

Brancaccio P, Maffulli N, Limongelli FM. Creatine kinase monitoring in sport medicine. Br Med Bull 2007;81-82:209-230.

Danielsson T, Carlsson J, Schreyer H, Ahnesjö J, Ten Siethoff L, Ragnarsson T, Tugetam $\AA$, Bergman P. Blood biomarkers in male and female participants after an Ironman-distance triathlon. PLoS One 2017;12: e0179324.

Fu F, Nie J, Tong TK. Serum cardiac troponin T in adolescent runners: effects of exercise intensity and duration. Int J Sports Med 2009;30:168172.

Jassal DS, Moffat D, Krahn J, Ahmadie R, Fang T, Eschun G, Sharma S. Cardiac injury markers in non-elite marathon runners. Int J Sports Med 2009;30:75-79.

Lee EC, Fragala MS, Kavouras SA, Queen RM, Pryor JL, Casa DJ. Biomarkers in sports and exercise: tracking health, performance, and recovery in athletes. J Strength Cond Res 2017;31:2920-2937.
Leischik R, Spelsberg N. Endurance sport and "cardiac injury": a prospective study of recreational ironman athletes. Int J Environ Res Public Health 2014;11:9082-9100.

Olcina G, Timón R, Brazo-Sayavera J, Martínez-Guardado I, Marcos-Serrano $\mathrm{M}$, Crespo $\mathrm{C}$. Changes in physiological and performance variables in non-professional triathletes after taking part in an Olympic distance triathlon. Res Sports Med 2018;26:323-331.

Park CH, Kim KB, Han J, Ji JG, Kwak YS. Cardiac damage biomarkers following a triathlon in elite and non-elite triathletes. Korean J Physiol Pharmacol 2014;18:419-423.

Stewart GM, Yamada A, Haseler LJ, Kavanagh JJ, Chan J, Koerbin G, Wood C, Sabapathy S. Influence of exercise intensity and duration on functional and biochemical perturbations in the human heart. J Physiol 2016;594:3031-3044.

Suzuki K, Peake J, Nosaka K, Okutsu M, Abbiss CR, Surriano R, Bishop D, Quod MJ, Lee H, Martin DT, Laursen PB. Changes in markers of muscle damage, inflammation and HSP70 after an Ironman Triathlon race. Eur J Appl Physiol 2006;98:525-534.

Thygesen K, Alpert JS, White HD; Joint ESC/ACCF/AHA/WHF Task force for the redefinition of myocardial infarction. Universal definition of myocardial infarction. J Am Coll Cardiol 2007;50:2173-2195.

Traiperm N, Gatterer H, Wille M, Burtscher M. Cardiac troponins in young marathon runners. Am J Cardiol 2012;110:594-598.

Whyte GP, George K, Sharma S, Lumley S, Gates P, Prasad K, McKenna WJ. Cardiac fatigue following prolonged endurance exercise of differing distances. Med Sci Sports Exerc 2000;32:1067-1072. 